\title{
Impact of droplets on liquid films in the presence of
}

\section{surfactant}

\author{
Zhizhao Che ${ }^{*, \dagger}$, and Omar K. Matar $*, \$$
}

State Key Laboratory of Engines, Tianjin University, Tianjin, 300072, China., and Department of Chemical Engineering, Imperial College London, London, SW7 2AZ, United Kingdom

E-mail: chezhizhao@tju.edu.cn; o.matar@imperial.ac.uk

\begin{abstract}
The impact of droplets on liquid films is ubiquitous in natural and industrial processes, and surfactants can significantly alter the impact process by changing the local surface tension. Here we study the impact of droplets on liquid films in the presence of surfactant using highspeed photography, and reveal the flow pattern by dye-tracing. The effects of the droplet size and speed, and the initial film thickness on the impact process are elucidated. The results show that the flow is significantly affected by adding surfactant to the droplet, the liquid film, or to both phases. In particular, the film dye patterns form concentric circles and flower-shaped structures at low and high droplet Weber numbers, respectively. We also show how surfactantinduced Marangoni stresses modify these flow patterns, and alter the characteristics of the phenomena associated with the impact process, such as the propagation of capillary waves, the evolution of the crown, and the formation of secondary droplets. During the impact of surfactant droplets on thin water films, the Marangoni stresses can be sufficiently strong so as to drive film de-wetting.
\end{abstract}

\footnotetext{
*To whom correspondence should be addressed

${ }^{\dagger}$ State Key Laboratory of Engines, Tianjin University, Tianjin, 300072, China.

†Department of Chemical Engineering, Imperial College London, London, SW7 2AZ, United Kingdom
} 


\section{Introduction}

The impact of droplets on liquid films is a common phenomenon in many natural processes and industrial applications. The outcomes of the impact process directly affect the performance of these applications or even cause catastrophic accidents, such as spray cooling of hot surfaces, icing on aircraft wings, and droplet-impact erosion on turbine blades. Despite the numerous studies in this area spanning more than a century, the droplet impact phenomenon is still far from being fully understood. ${ }^{1-3}$ Many outstanding questions are still attracting attention, such as the origin of splashing, ${ }^{4,5}$ the evolution of the air layers below the impacting droplets, ${ }^{6,7}$ and the entrapment of air bubbles. ${ }^{8,9}$ The impact process is complicated by many controlling parameters, such as the properties of the fluids (e.g., surface tension, viscosity, density, and rheology ${ }^{10}$ ), the parameters of impact (e.g., droplet speed, impact angle, and film velocity ${ }^{11,12}$ ), and the ambient condition (e.g., temperature ${ }^{13}$ and pressure ${ }^{14}$ ). A range of phenomena may occur depending on the relative magnitude of these parameters, such as bouncing, ${ }^{15,16}$ partial coalescence, ${ }^{17-19}$ "crown" splashing, ${ }^{20-22}$ and "prompt" splashing. ${ }^{23,24}$

Extensive efforts and rapid progress have been made in the study of droplet impact over the last two decades. ${ }^{11,25-33}$ The development of high-speed photography in particular allows us to study the impact process with a high temporal resolution. ${ }^{34}$ Various new techniques have also been introduced to unveil the complex droplet morphologies and flow structures during the impact process, e.g., X-ray phase-contrast imaging, ${ }^{35}$ ultrafast interference imaging, ${ }^{7}$ brightness based laser induced fluorescence, ${ }^{36}$ and modified micro-particle tracking velocimetry. ${ }^{37}$

The presence of surfactant, either as an additive or a contaminant, can alter the interfacial dynamics through its local reduction of the surface tension in high surfactant concentration regions; gradients of surface tension then give rise to Marangoni stresses that drive flow in the direction of higher surface tension. The effect of surfactant on the dynamics of droplets impacting a solid substrate has been investigated ${ }^{38,39}$ and found to inhibit droplet bouncing and column breakup in the case of surfactant-laden droplets on hydrophobic surfaces. ${ }^{39}$ Crucially, surfactants also affect the process of droplet coalescence: during the coalescence of a surfactant-free droplet with a 
surfactant-laden one, the Marangoni effect gives rise to a "mushroom-like" structure in the internal flow, which has been observed experimentally in a recent study. ${ }^{40}$

Although many factors affecting the impact process have been investigated, the effect of surfactantinduced Marangoni flow during the impact process on liquid films has not yet been explored, to the best of our knowledge. To study this effect, four impact configurations are considered here ranging from surfactant-free water droplets and films, to surfactant-laden ones, as detailed in the

experimental section below. Blanchette et al. ${ }^{41,42}$ studied the coalescence of a droplet with a liquid reservoir of a miscible fluid of different surface tension at a negligible droplet initial velocity, and observed the ejection of droplets from the top of the primary droplet. When the liquid reservoir is sufficiently thin, i.e., of the order of the droplet diameter, at most, which is the case considered here, both the liquid film and the underlying substrate participate in the impact process. The substrate confines the flow of the liquid during the coalescence process, and results in unique features of droplet morphology. In addition, in the present study, we examine situations in which the droplet speed is appreciably large, and inertia plays an important role in the impact dynamics, which further distinguishes the present work from previous studies. ${ }^{41,42}$

The rest of this paper is organised as follows. The experimental details, including the setup and the data-collection procedure, are described in the next section. Then, the results are discussed, including the impact outcomes, the flow pattern in the film following the impact, and the effects of the droplet speed, the average film thickness, and the droplet size in the different configurations defined above are analysed.

\section{Experimental}

The experiment setup is shown in Figure 1a. Droplets were generated from the tips of blunted syringe needles (Adhesive dispensing Ltd, UK). The size and the speed of the droplets were varied by changing the size of the needle and its height above the film. The films were prepared by depositing a liquid layer in a glass petri dish with an inner diameter of $100 \mathrm{~mm}$ (Scientific 
(a)
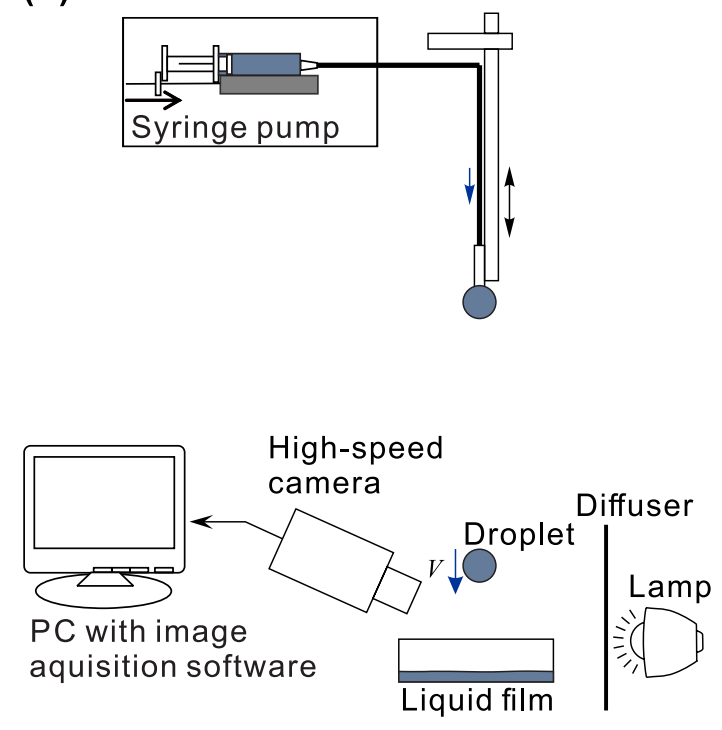

(b)
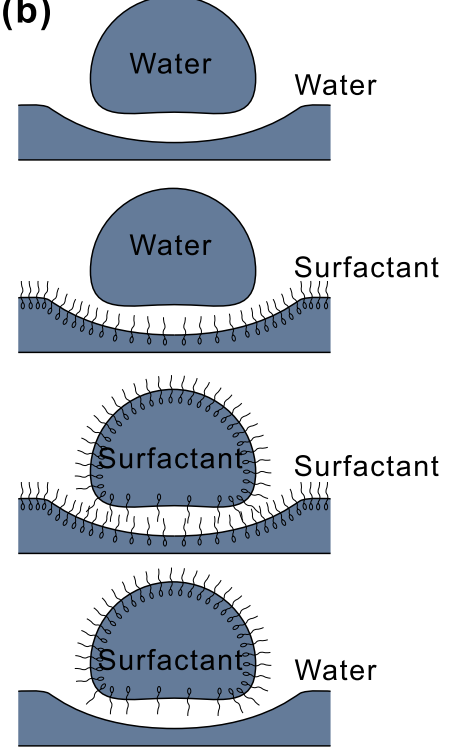

Figure 1: (a) Schematic diagram of the experimental setup; (b) the four configurations of droplet impact on liquid films studied in the present work, i.e., water droplets on water films ("waterwater"), water droplets on surfactant-laden films ("water-surfactant"), surfactant-laden droplets on surfactant-laden films ("surfactant-surfactant"), and surfactant-laden droplets on surfactant-free water films ("surfactant-water").

Laboratory Supplies, UK); the film thickness was varied by changing the film volume.

A high-speed camera (Olympus i-SPEED 3) was used to record the impact process at 10,000 frames per second with a macro lens (Nikon AF Micro-Nikkor $60 \mathrm{~mm} \mathrm{f} / 2.8 \mathrm{D}$ ) at a resolution of $528 \times 396$ pixels. The camera was placed at an angle of $44^{\circ}$ to the horizontal to obtain flow information of both the crown and the pattern after the impact. This arrangement allows us to achieve a resolution of $46 \mu \mathrm{m} / \mathrm{pixel}$ and $67 \mu \mathrm{m} / \mathrm{pixel}$ in the horizontal and vertical directions, respectively. A halogen lamp with a diffuser was used for illumination. The high-speed images were processed using a customised, in-house Matlab programme.

Four different configurations were considered, as shown in Figure 1b: surfactant-free water droplets impacting on surfactant-free water films (termed "water-water" systems), surfactant-free water droplets impacting on surfactant-laden films ("water-surfactant"), surfactant-laden droplets impacting on surfactant-laden films ("surfactant-surfactant"), and surfactant-laden droplets impacting on surfactant-free water films ("surfactant-water").

De-ionised water (density $\rho=10^{3} \mathrm{~kg} / \mathrm{m}^{3}$, viscosity $\mu=10^{-3} \mathrm{~Pa} \cdot \mathrm{s}$ at room temperature) 
was used as the working fluid, and sodium dodecyl sulphate (SDS, Sigma-Aldrich, UK) as the surfactant. SDS is a water-soluble surfactant with a critical micelle concentration (CMC) of 8.2 $\mathrm{mM}$, and it is used at a concentration of $65.6 \mathrm{mM}$ in water. The surface tension (measured by a Wilhelmy Plate tensiometer, Attension Sigma 701), before and after the addition of surfactant, was $71.2 \mathrm{mN} / \mathrm{m}$ and $34.4 \mathrm{mN} / \mathrm{m}$, respectively. To distinguish between the liquid in the droplet and the film, a dye (Nigrosin water soluble, VWR, UK) was added to the droplet at a concentration of $0.25 \%$ by weight to reveal the flow pattern in some cases. At such a low concentration of the dye, the change in viscosity and surface tension was negligible, which was confirmed via direct measurement of the viscosity (using an Anton Paar MCR 302 rheometer) and the surface tension (using an Attension Sigma 701 tensiometer).

The key parameters varied in this study include the droplet speed, $v$, the droplet diameter, $d$, and the film thickness, $h$, in the ranges $0.24 \sim 2.72 \pm 0.03 \mathrm{~m} / \mathrm{s}, 1.83 \sim 3.94 \pm 0.02 \mathrm{~mm}$, and $0.3 \sim 3.0 \pm 0.01 \mathrm{~mm}$, respectively, in addition to the presence of surfactant in either the droplet or film phases, or both. The magnitude of the relevant dimensionless groups resulting from this range of parameters is shown in Table 1: the Weber number, We, measures the relative importance of inertia and surface tension, the Ohnesorge number, Oh, the ratio of viscous to inertial and surface tension forces, and the Bond number, Bo, the ratio of gravitational to surface tension forces. In addition, we define a dimensionless film thickness, $\hat{h}$, a ratio of the average film thickness and the droplet diameter, and a dimensionless surface tension difference, $\hat{\sigma}$, a ratio of the difference in drop and film surface tension, normalised by the surface tension of surfactant-free water.

\section{Results and Discussion}

We begin the presentation of our results by examining the case wherein a droplet of water impacts a surfactant-free water film. In the parameter ranges studied in the present work, several outcomes were observed including bouncing, partial coalescence, total coalescence, and "prompt" and "crown" splashing, as shown in Figure 2. Since these outcomes have been well documented in the literature, 
Table 1: Dimensionless groups governing the impact dynamics.

\begin{tabular}{lll}
\hline Dimensionless number & Definition & Range \\
\hline Bond number & Bo $=\frac{\rho g d^{2}}{\sigma_{\text {drop }}}$ & $0.72 \sim 2.7$ \\
Dimensionless film thickness & $\hat{h}=h / d$ & $0.076 \sim 1.6$ \\
Dimensionless surface tension difference & $\hat{\sigma}=\frac{\sigma_{\text {drop }}-\sigma_{\text {film }}}{\sigma_{\text {water }}}$ & $-0.52,0,0.52$ \\
Ohnesorge number & $\mathrm{Oh}=\frac{\mu}{\sqrt{\rho \sigma_{\text {drop }}}}$ & $0.0019 \sim 0.0040$ \\
Weber number & $\mathrm{We}=\frac{\rho v^{2} d}{\sigma_{\text {drop }}}$ & $1.9 \sim 664$ \\
\hline
\end{tabular}

they are only discussed briefly here in order to establish a baseline for comparison with the droplet impact dynamics involving surfactants. The interested readers are referred to the literature for more details. ${ }^{11,25-33}$

At a low impact speed, characterised by small We numbers, the droplet can rebound from the film surface, leaving a dimple on the film, which produces a capillary wave that propagates radially outwards from the impact point (see Figure 2a). The rebound is caused by an air layer formed during the impact process between the droplet and the liquid film, ${ }^{6,7,11}$ which undergoes drainage as the droplet approaches the film. If the air layer ruptures during drainage, then merging of the droplet with the film occurs, resulting in either partial or total coalescence. In the case of partial coalescence, a small droplet is produced from the apex of the primary droplet due to propagation of strong capillary waves formed at the instant of the rupture of the gas layer (see Figure 2b). In contrast, for total coalescence, no small droplet is produced, and the capillary waves are damped out as they propagate on the liquid film (see Figure 2c).

If the impact speed is high, corresponding to large We numbers, two types of droplet splashing may take place: "prompt" and "crown" splashing. In prompt splashing, the high-speed droplet generates a thin liquid sheet from the neck that connects the droplet to the liquid film immediately following contact; the liquid sheet then breaks up into tiny droplets during its extension outward in the air (see Figure 2d). In crown splashing, a crown is formed first following impact, and then droplets are shed from the rim of the crown (see Figure 2e). 


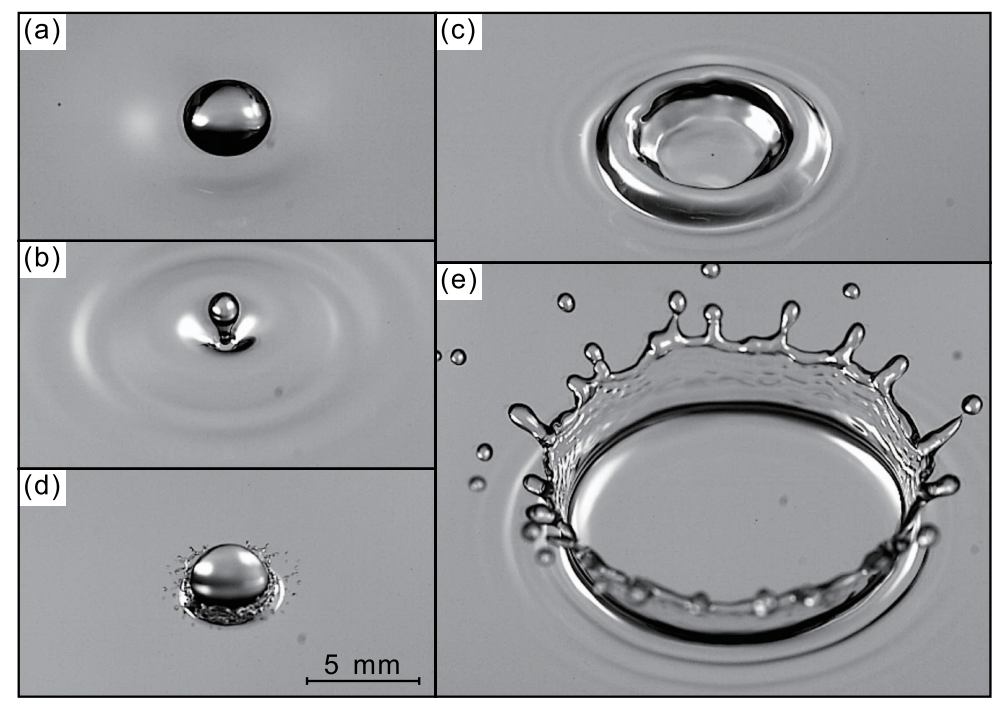

Figure 2: Impact outcomes of water droplets on water films: (a) bouncing, (b) partial coalescence, (c) total coalescence, (d) prompt splashing, and (e) crown splashing. The droplet diameter is $d=3.15 \mathrm{~mm}$, and the film thickness and the droplets speed are (a) $h=1.5 \mathrm{~mm}, v=0.24 \mathrm{~m} / \mathrm{s}$, (b) $h=3.0 \mathrm{~mm}, v=0.24 \mathrm{~m} / \mathrm{s}$. (c) $h=0.7 \mathrm{~mm}, v=0.91 \mathrm{~m} / \mathrm{s}$, (d) $h=3.0 \mathrm{~mm}, v=2.7 \mathrm{~m} / \mathrm{s}$, (e) $h=0.3$ $\mathrm{mm}, v=2.7 \mathrm{~m} / \mathrm{s}$, respectively. The dimensionless impact parameters are $\mathrm{Oh}=0.0021, \hat{\sigma}=0$ and (a) $\hat{h}=0.48$, We $=2.6$; (b) $\hat{h}=0.95$, We $=2.6$; (c) $\hat{h}=0.22, \mathrm{We}=37$; (d) $\hat{h}=0.95$, We $=328$; (e) $\hat{h}=0.095$, We $=328$, respectively.

\section{Surfactant-laden droplet}

For impact processes with surfactants, surface tension gradient may appear. The magnitude of the surface tension difference can be estimated from dimensionless surface tension difference $\hat{\sigma}$, and its relative importance with gravitational, inertial, and viscous forces can be estimated by combining $\hat{\sigma}$ with Bo, We, or Oh respectively. The surface tension gradient varies with time and space, and is complicated by the absorption/desorption of surfactant molecules at the interface and the formation of micelles.

We now turn our attention to situations in which the surfactant has been added to the droplet phase only. As a result of this, the impact dynamics is altered significantly, which can be seen from the patterns of dye during the impact process (see Figure 3a-b), and those in the surfactantsurfactant configuration for comparison (see Figure 3c-d).

In the surfactant-surfactant configuration, at a low impact speed (small We), the relative effect of surface tension is large, while the effect of inertial forces is small. Therefore, circular dye 


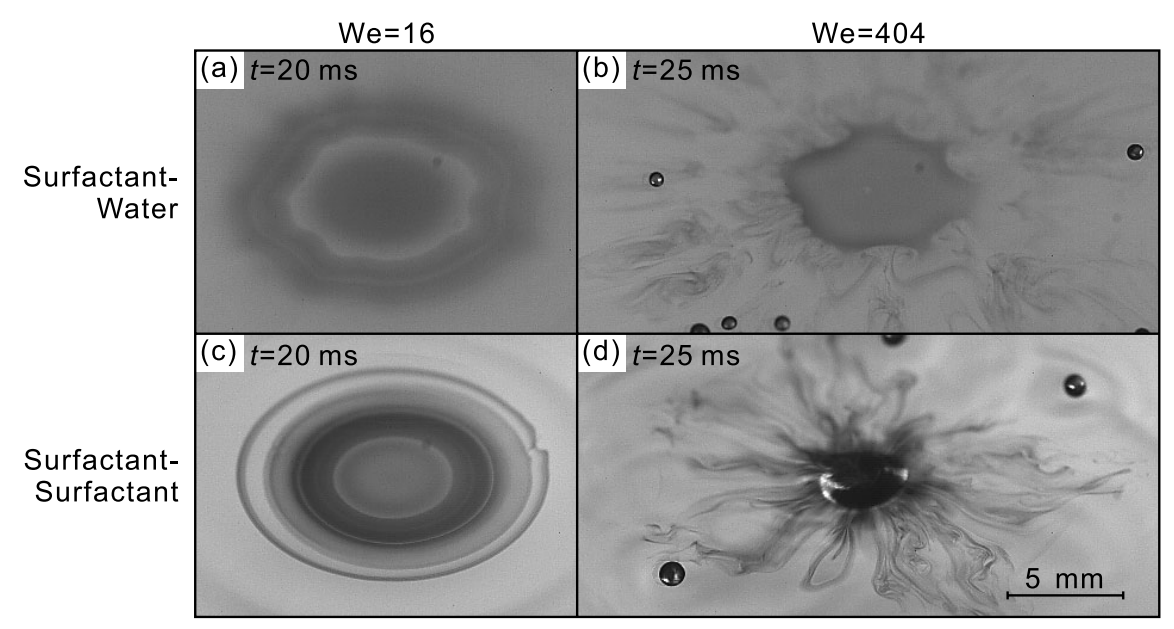

Figure 3: Flow pattern in the liquid film after the impact of droplets revealed by the propagation of droplet fluid with dye. (a-b) Impact of surfactant droplets on water films; (c-d) impact of surfactant droplets on surfactant films. The droplet diameter is $d=2.47 \mathrm{~mm}$, the film thickness is $h=0.3$ $\mathrm{mm}$, and the droplet speeds are (a, c) $v=0.47 \mathrm{~m} / \mathrm{s}$ and $(\mathrm{b}, \mathrm{d}) v=2.4 \mathrm{~m} / \mathrm{s}$, respectively. The impact dimensionless parameters are $\hat{h}=0.12, \mathrm{Oh}=0.0034$, and (a) $\mathrm{We}=16, \hat{\sigma}=-0.52$; (b) $\mathrm{We}=404$, $\hat{\sigma}=-0.52$; (c) $\mathrm{We}=16, \hat{\sigma}=0$; (d) $\mathrm{We}=404, \hat{\sigma}=0$, respectively. Movies for these processes are available as Supporting Information (Videos 1-4).

patterns form in the liquid film after the impact of the droplet (see Figure 3c). Several concentric rings are shown in the dye pattern, indicating a non-uniform distribution of the droplet fluid after the impact. Since the diffusion of dye in such a short instant can be negligible compared to the convection of the dye fluid, darker rings indicate thicker droplet fluid, and the outer boundary of the dark ring indicates the propagation precursor of the droplet fluid. Following the impact, there is a strong radial flow of the droplet fluid outwards, producing a strong wave with large amplitude (the wave that develops to the crown at high impact speeds). Therefore, unlike surface waves without net flow in many deep water problems, the fluid elements here in the thin liquid layer no longer return to their original position due to the radial flow and the shear effect of the substrate. ${ }^{43}$ The front of the wave has larger radial velocity, while the other region has lower radial flow velocity. Therefore, the different radial velocity stretches the droplet fluid to different thicknesses. As a consequence, the droplet fluid has different thicknesses in different radial positions, resulting concentric rings in the liquid film. At high impact speeds (high We), flower patterns of dye appear in the liquid film (see Figure 3d). Inertia also becomes important at high speeds, and 
the propagation of the droplet fluid in the liquid film after the coalescence is unstable because the viscous effects that could potentially stabilize the flow are negligible. Therefore, instability develops as the droplet liquid penetrates into the film and forms the flower-patterned flow structure. This is in agreement with the flower-patterned film thickness found previously. ${ }^{27}$

In contrast, in the surfactant-water configuration, upon coalescence of the droplet with the liquid film, a rapid radial flow occurs due to radial surface tension gradients: the presence of surfactant in the droplet results in a low surface tension region at the impact point, while regions far from it in the liquid film have a higher surface tension. The resulting surface tension gradient drives Marangoni flow towards regions of high tension, which leads to blurring of the dye patterns in the film, as shown in Figure 3a-b. The time-scale associated with Marangoni flow in the liquid film $t_{M}$ can be found by considering the balance between the Marangoni stress and the viscous stress. The Marangoni stress on the surface of the liquid film scales as $\frac{\partial \sigma}{\partial r} \sim \frac{\Delta \sigma}{d}$, and the shear stress in the liquid film scales as $\mu \frac{\partial u}{\partial z} \sim \mu \frac{V_{\text {film }}}{h} \sim \mu \frac{d}{h t_{M}}$, where $V_{\text {film }}$ is the characteristic velocity in the liquid film $V_{\text {film }} \sim d / t_{M}$. The balance of the Marangoni stress and the shear stress gives $t_{M}=\mu \frac{\mu d^{2}}{h \Delta \sigma}$. For the parameter values that characterise these experiments, $t_{M}$ is of order $10^{-4} \mathrm{~s}$. This is comparable to the droplet impact time-scale, $10^{-4} \sim 10^{-2} \mathrm{~s}$, indicating that Marangoni flow plays a key role in the dynamics.

The gradient of the surfactant concentration also accelerates the propagation of the capillary waves, as shown in Figure 4. In comparison with the impact without surface tension gradients (i.e., the "water-water" configuration), the capillary wave propagation in the surfactant-laden case is faster due to the presence of the Marangoni stresses on the surface of the liquid film. Even though the clean water droplet and the surfactant-laden droplet in Figure 4 are not identical in size, the droplet size is expected to play only a minor role on the propagation of capillary wave. This is because the propagation of capillary wave on the liquid film occurs at the later stage of the impact and it is mainly determined by film thickness, surface tension, fluid viscosity, and fluid density.

For sufficiently small average film thicknesses, the radial Marangoni flow is sufficiently strong so as to drive severe film thinning such that intermolecular forces become operational leading to 


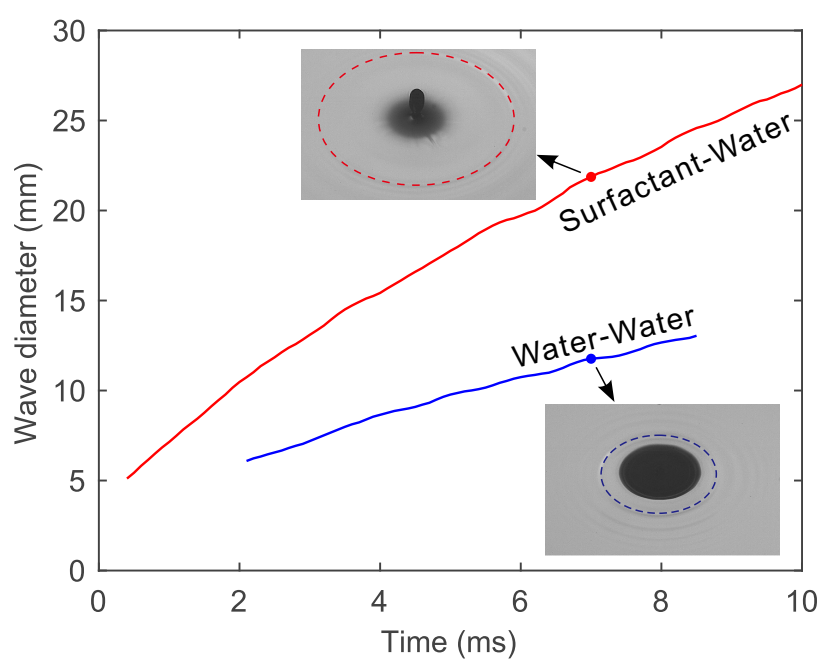

Figure 4: Temporal evolution of the diameter of capillary waves resulting from droplet impact. The red line represents the impact of a surfactant-laden droplet on a surfactant-free water film $(v=0.24$ $\mathrm{m} / \mathrm{s}, h=0.3 \mathrm{~mm}, d=2.47 \mathrm{~mm}, \mathrm{We}=3.1, \hat{h}=0.12, \mathrm{Oh}=0.0034)$, and the blue line represents the impact of a surfactant-free water droplet on a surfactant-free water film $(v=0.24 \mathrm{~m} / \mathrm{s}, h=0.3 \mathrm{~mm}$, $d=3.15 \mathrm{~mm}, \mathrm{We}=2.6, \hat{h}=0.095, \mathrm{Oh}=0.0021)$. The snapshots at $t=7 \mathrm{~ms}$ after the coalescence are shown and the capillary waves are highlighted by dashed lines.

film dewetting; this is shown in Figure 5. The thinning process is resisted by viscous forces which are inversely proportional to the square of the local thickness. Film thinning is also counter-acted by gravitational forces, whose relative importance to the surface tension force can be estimated by $B o \times \hat{h}^{2}$ since the characteristic length scale here should be the film thickness instead of the droplet diameter. For sufficiently long time, the spreading of surfactant which accompanies the impact process also leads to a decrease in the magnitude of the Marangoni flow responsible for film thinning, and an increase in the local surface tension which promotes film healing. The healing of the liquid film is driven by the gravity, whose relative magnitude is indicated again by $B o \times \hat{h}^{2}$. The dewetting of the liquid film results in non-uniform film thickness and gravity plays the role when Maragnoni flow is negligible and flattens the film finally. ${ }^{44}$ The time-scale of film healing can be estimated from $t_{\text {healing }} \sim L / \sqrt{g h} \sim 1 \mathrm{~s}$ where $L$ is the characteristic length of the capillary wave diameter. 


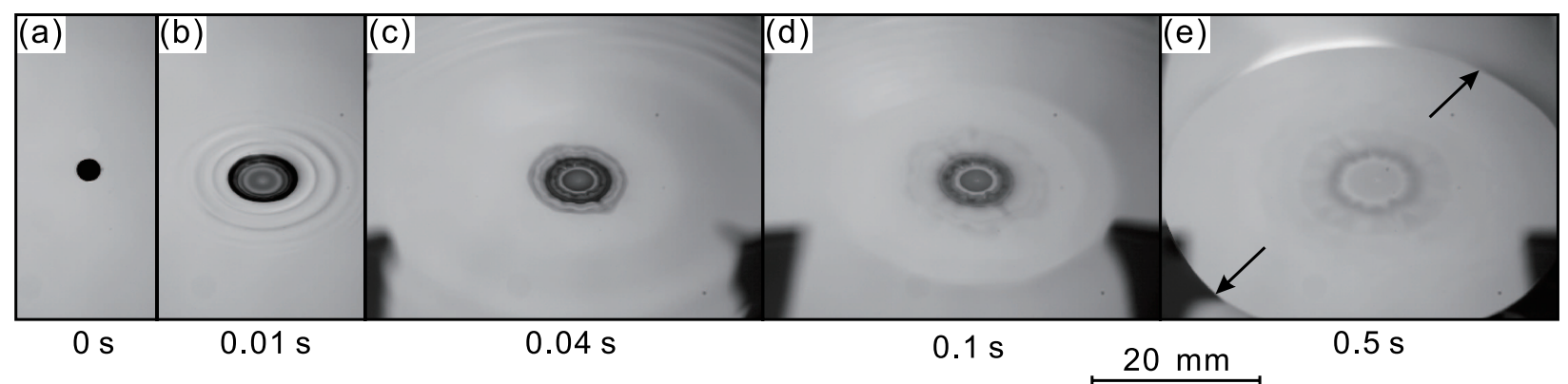

Figure 5: De-wetting of the water film upon the impact of a surfactant droplet. The droplet size, the droplet speed, and the film thickness are $d=2.47 \mathrm{~mm}, v=0.91 \mathrm{~m} / \mathrm{s}, h=0.7 \mathrm{~mm}$, respectively. The dimensionless impact parameters are $\hat{h}=0.28, \mathrm{Oh}=0.0034$, We $=60$, and $\hat{\sigma}=-0.52$, respectively. The line of de-wetting is highlighted by arrows.

\section{Surfactant-laden liquid film}

We now examine the effect of adding the surfactant to the liquid film but not the droplet. As shown in Figure 6, the dynamics are characterised by qualitatively different behaviour depending on the droplet Weber number. For We $=2.6$, the impact process results in droplet bouncing, which is promoted by the presence of surfactant on the surface of the film. Upon the impact of the droplet, during the drainage of the air layer separating the droplet and the film, the outward flow of air and liquid film causes the surface area of the surfactant-laden film extends, reducing the local surface concentration of the surfactant since the diffusion of surfactant molecules from the bulk to the surface is not rapid enough to replenish. A lower concentration corresponds to a higher surface tension, and results in a Marangoni stress to counteract the drainage of the air. Therefore, surfactant has an effect to rigidify the film surface and promote droplet bouncing. Similar effects of surfactants to retard the thinning of liquid films between droplets or between a droplet and a solid surface have been reported. ${ }^{45-47}$

The bouncing of droplets on surfactant-laden film is in contrast to the water-water case in which droplet impact at low Weber numbers results in coalescence, as shown in Figure 6a and Figure 6f. When We is increased from 2.6 to 19 , the droplet coalesces with the liquid film upon the contact for both the water-water, and water-surfactant cases; the capillary waves produced following coalescence in the latter case, however, have a slower propagation speed than those 


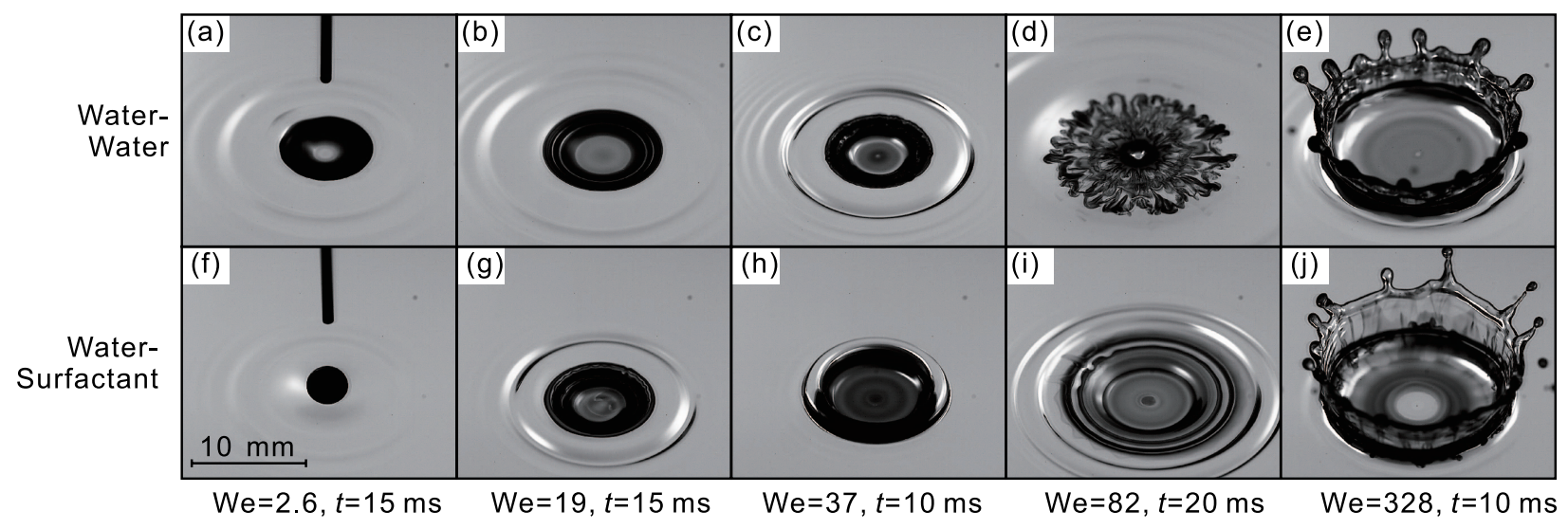

Figure 6: Comparing between the impact of water droplets on water films and on surfactant films. The droplet size and the film thickness are $d=2.47 \mathrm{~mm}$ and $h=0.7 \mathrm{~mm}$. (a, f) $v=0.24 \mathrm{~m} / \mathrm{s}$, $\mathrm{We}=2.6$. The droplet is easier to bounce on the surfactant film; (b, g) $v=0.65 \mathrm{~m} / \mathrm{s}, \mathrm{We}=19$. The surfactant liquid film results in capillary waves with higher magnitudes and a slower propagation speed due to the lower surface tension; $(\mathrm{c}, \mathrm{h}) v=0.91 \mathrm{~m} / \mathrm{s}$, We $=37$. During the impact on the surfactant film, the capillary wave is strong and has a crown shape, which is not observed during the impact on the water film. $(\mathrm{d}, \mathrm{i}) v=1.4 \mathrm{~m} / \mathrm{s}, \mathrm{We}=82$. Crowns form in both cases, and the resultant pattern of dye on the surfactant film is more regular than that on the water film, indicating a more stable flow in the surfactant film. $(\mathrm{e}, \mathrm{j}) v=2.7 \mathrm{~m} / \mathrm{s}$, We $=328$. The droplets splash in both cases, and more secondary droplets are generated during the impact on surfactant films than that on water films, and the crown is thinner and survives longer. The other dimensionless impact parameters are $\mathrm{Oh}=0.0021, \hat{h}=0.22$, and (a-e) $\hat{\sigma}=0$, (f-j) $\hat{\sigma}=0.52$, respectively. Movies for (d) and (i) are available as supporting information (Videos 5-6).

associated with the water-water case. This is as expected since capillary wave speed is proportional to the surface tension which is lower in the surfactant-laden liquid film case.

If We is increased further, the amplitude of the capillary waves on the surfactant-laden liquid film is significantly larger than the initial film thickness, so the wave has a crown shape; this is not present in the water-water case at this value of the Weber number (see Figure 6c and Figure 6j). Although crowns are formed in both the water-water and water-surfactant cases for We $=82$, following the dissipation of the crown, concentric circles of dye form in the surfactant film, while flower patterns of dye form in the water film. This is again due to the interfacial rigidification brought about by the presence of surfactant which promotes Marangoni stresses that resist interfacial deformation. At even larger Weber numbers $(\mathrm{We}=328)$, splashing happens both on water films and on surfactant films. Compared to the splashing on surfactant-free water films, however, the splashing on surfactant-laden films produces a thinner and higher crown sheet, and 
generates more secondary droplets with smaller sizes. The latter observation is due to the thinner crown generated after the impact on surfactant-laden film than that on the surfactant-free film. The thinner crown then produces more, and smaller, secondary droplets driven by a Rayleigh-Taylor instability. $^{22}$

\section{Effect of the Weber number}

The crown dynamics are now examined for the four configurations shown in Figure 7a. Here, the Weber number is varied parametrically, starting from values that exceed the threshold We necessary for crown-formation in all configurations including water-water systems. It is clear from Figure $6 \mathrm{a}$ that the size of the crown increases with We, and that secondary droplets are formed from the rim of the crown at sufficiently high We values. To compare quantitatively the propagation of the crown during the impact process for the different configurations, the temporal evolution of the diameter of the crown rim is plotted in Figure $7 \mathrm{~b}$. The rim of the crown was obtained by image processing in Matlab, and its diameter is obtained by linear square fitting of the rim. As expected, a higher We results in a faster crown propagation in all configurations; this is due to the higher inertia, which is the driving force for crown formation. It is also evident from Figure 7 that the capillary wave speed is larger for surfactant-water than for surfactant-surfactant systems; indeed, the film beyond the crown is very smooth in the surfactant-surfactant case.

\section{Effect of film thickness}

The average film thickness is an important parameter affecting the impact process. In Figure 8, we show the outcome of varying the average film thickness, for fixed droplet speed, for all four configurations. For water droplets impacting on thin water films, the crown is thin and high, and disintegrates rapidly, with many secondary droplets generated from its rim. With increasing film thickness, the crown thickness increases and its height decreases; consequently, fewer or even no secondary droplets are generated than in the lower film thickness case. For a thick water film, a cavity on the liquid film is generated after the impact, and it is due to the reduced confinement 

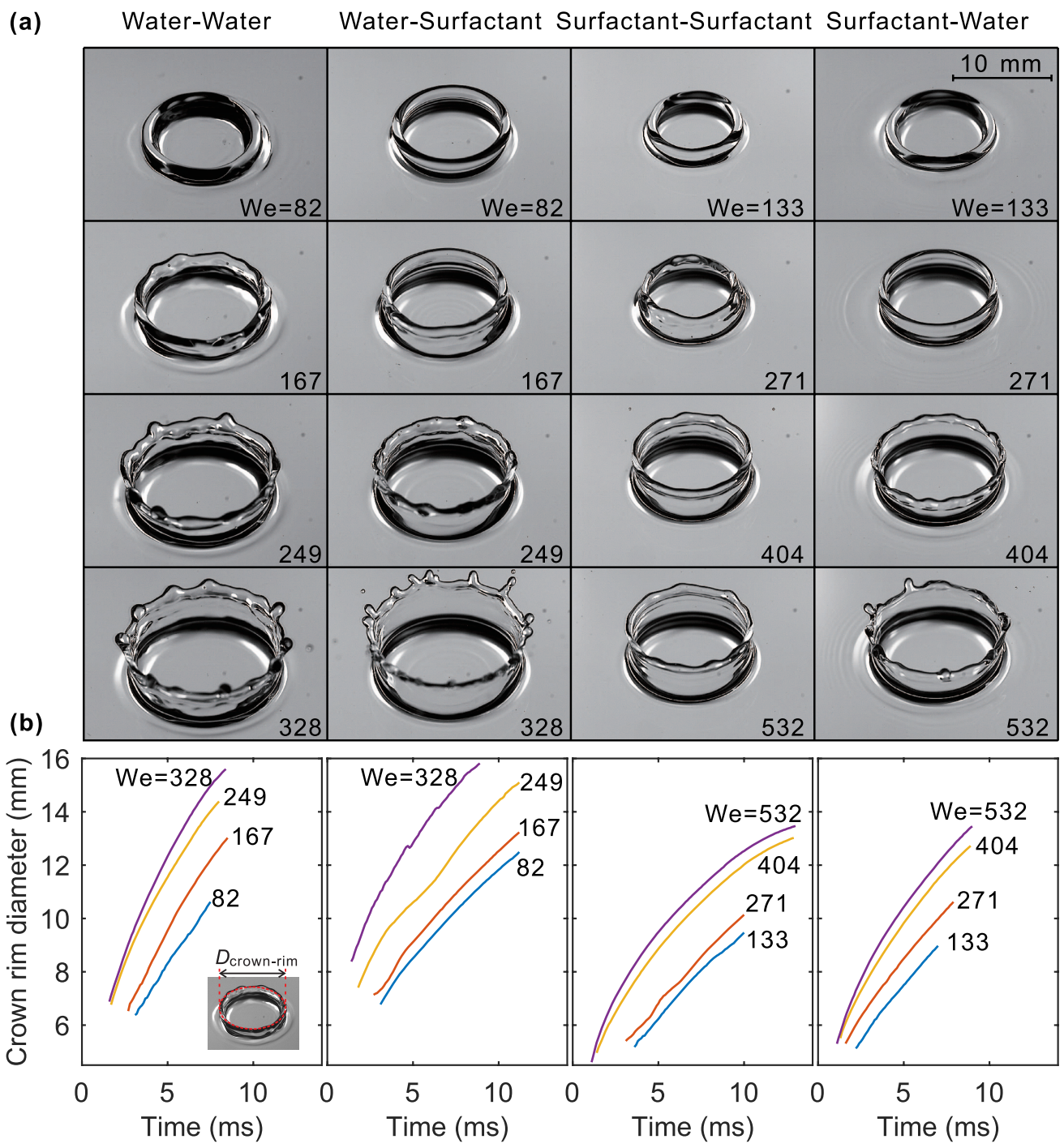

Figure 7: Effect of the Weber number on impact process. (a) Snapshots of the crown formed during the impact at $t=7 \mathrm{~ms}$; (b) evolution of the horizontal diameters of the crown rims. The droplet speeds are $1.4,1.9,2.4$, and $2.7 \mathrm{~m} / \mathrm{s}$, respectively. The diameters of water and surfactant droplets are $d=3.15 \mathrm{~mm}$ and $d=2.47 \mathrm{~mm}$, respectively, and the film thickness is $h=0.7 \mathrm{~mm}$. For the water-water and water-surfactant configurations, $\hat{h}=0.22, \mathrm{Oh}=0.0021$. For the surfactantsurfactant and surfactant-water configurations, $\hat{h}=0.28, \mathrm{Oh}=0.0028$. 

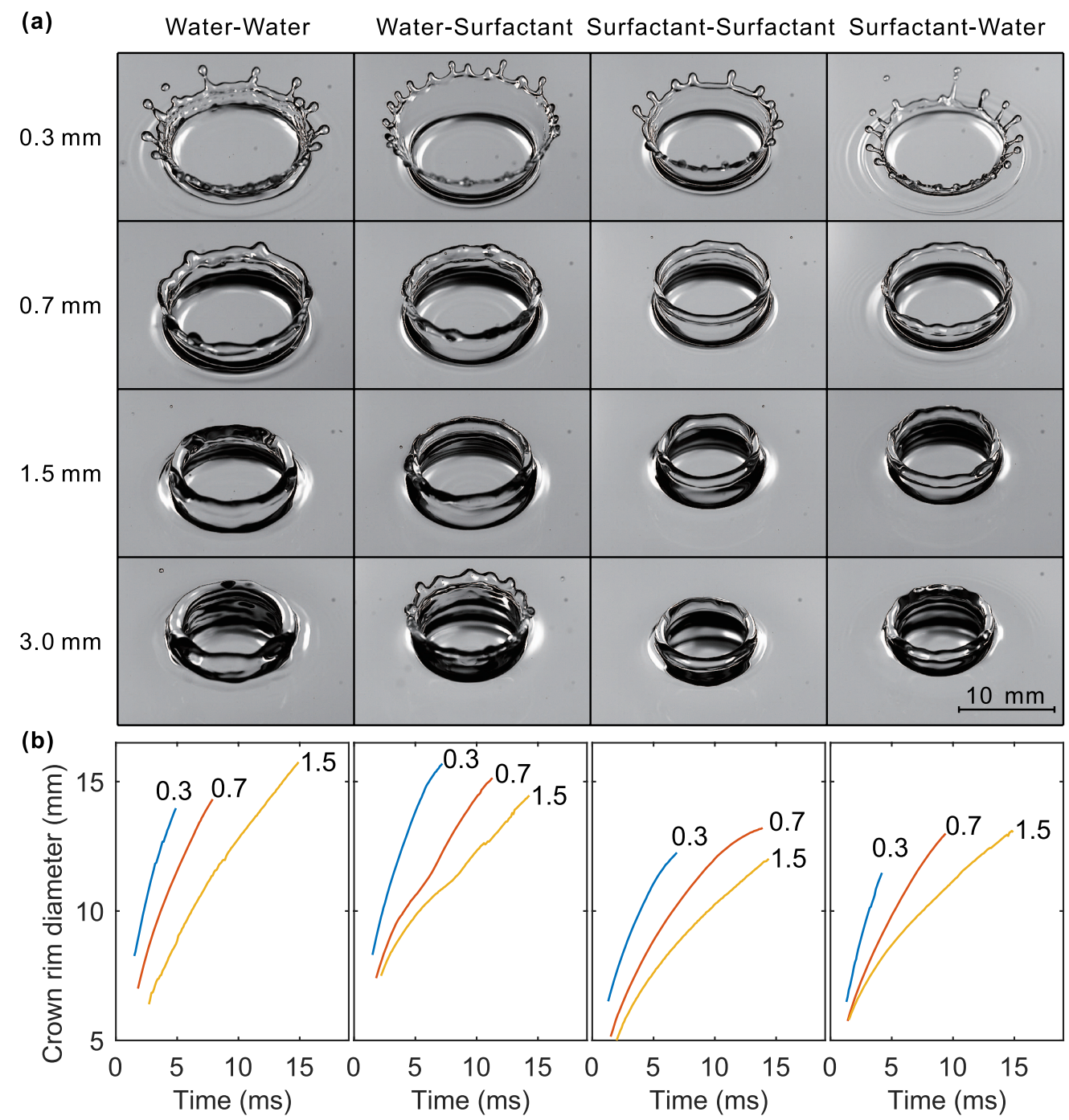

Figure 8: Effect of the film thickness on impact process. (a) Snapshots of the crown formed during the impact at $t=7 \mathrm{~ms}$, (b) evolution of the horizontal diameters of the crown rims. The numbers next to the curves are the thicknesses of the liquid films in $\mathrm{mm}$. The diameters of water and surfactant droplets are $d=3.15 \mathrm{~mm}$ and $d=2.47 \mathrm{~mm}$, respectively, and the droplet speed is $v=2.4 \mathrm{~m} / \mathrm{s}$. The crown rim diameters at $h=3.0 \mathrm{~mm}$ are not plotted in (b) because what the impact produces are more like cavities than crowns. For the water-water and water-surfactant configurations, $\mathrm{We}=249, \mathrm{Oh}=0.0021$, and the dimensionless film thickness $\hat{h}$ from top to bottom are $0.095,0.22,0.48,0.95$, respectively. For the surfactant-surfactant and surfactant-water configurations, $\mathrm{We}=404, \mathrm{Oh}=0.0034$, and the dimensionless film thickness $\hat{h}$ from top to bottom are $0.12,0.28,0.60,1.2$, respectively. 
effect of the substrate. Therefore, the droplet fluid, upon impact can flow both downward and outward to produce a cavity in the liquid film. In contrast, for the impact of droplets on thin liquid films, the confinement of the substrate only allows the droplet fluid to flow outwards to form a crown. Regarding the propagation speed of the crown, the crown formed on thinner films grows faster than that on thick films (see Figure $8 \mathrm{~b}$ ). This is because the droplet fluid needs to overcome the resistance of the liquid film to form the crown. A thick liquid film indicates a larger inertia and consequently a larger resistance. This trend is similar for surfactant-laden droplets impacting on surfactant-laden liquid films (third column of Figure 8).

For water droplets impacting on thin surfactant-laden films (second column of Figure 8), the crown is also thin and high, but it does not disintegrate as rapidly as in the water-water case. This is because of the lower surface tension of the surfactant-laden liquid film, which can enhance the stability of the crown. For surfactant droplets impacting on water films (fourth column of Figure 8), the crown also disintegrates rapidly at a thin film thickness, while the crown on surfactant-laden films (third row of Figure 8) has a longer life time. This is due to the crown produced from the water film having a higher surface tension, which can destabilise the surface of the crown and lead to the formation of secondary droplets. For sufficiently thick films, the difference in flow dynamics between the various configurations reduces mainly because the crown-generation process on thicker films is dominated by inertia with surface tension effects playing only a minor role.

\section{Effect of droplet size}

The effect of the droplet size on the impact process is studied by changing the diameters of the syringe tips. Larger droplets are formed from larger syringe tips, and in the splashing regime, they generate larger crowns and produce more secondary droplets (see Figure 9a). As the droplet size decreases, the kinetic energy of the droplet reduces, and the crown becomes small and very

stable, which essentially eliminates the formation of secondary droplets. This is supported by the temporal evolution of the crown diameter curves shown in Figure $9 \mathrm{~b}$ for the various configurations. 

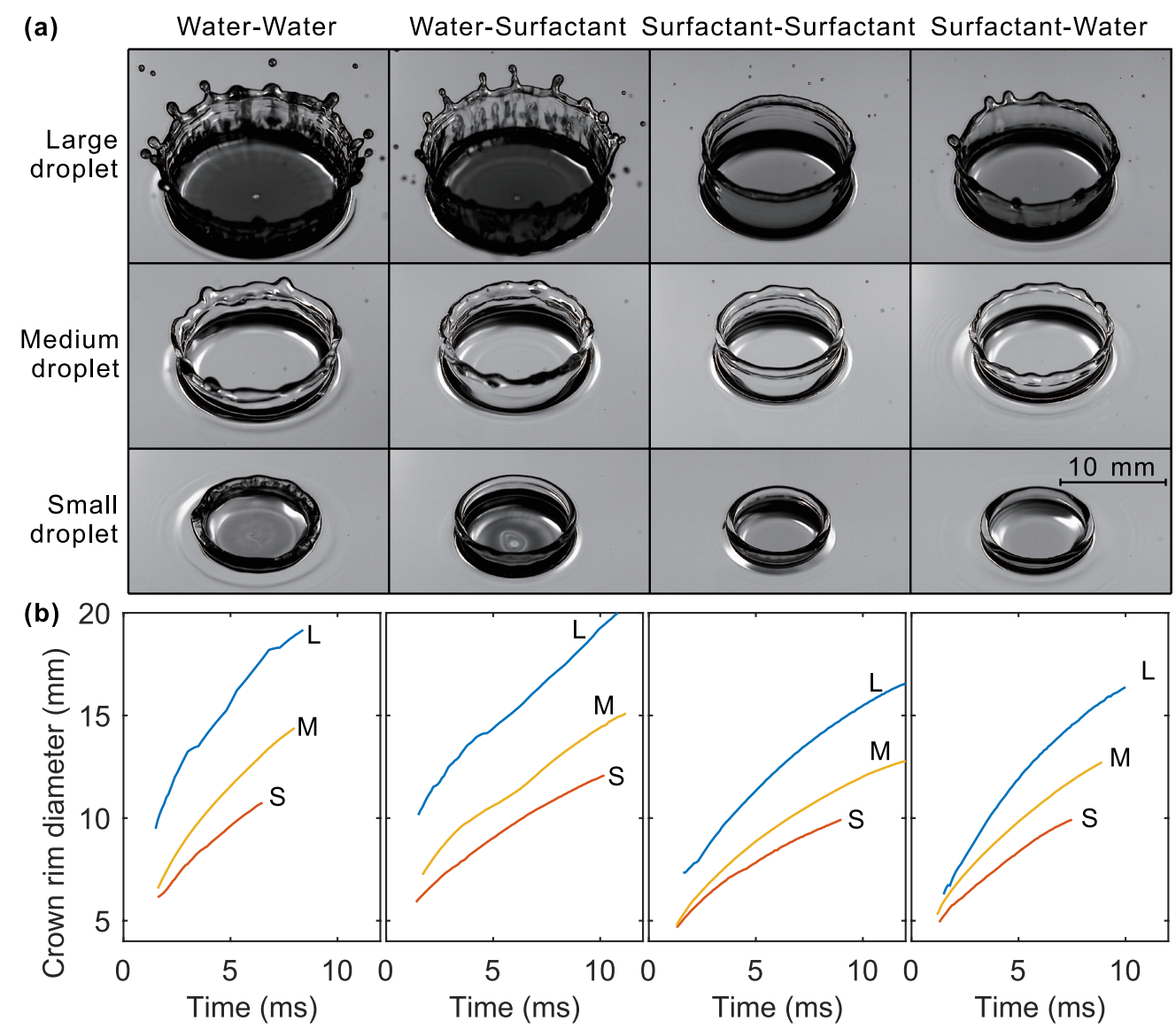

Figure 9: Effect of the droplet diameter on impact process. (a) Snapshots of the crown formed during the impact at $t=7 \mathrm{~ms}$, (b) evolution of the horizontal diameters of the crown rims. The diameters of the large (L), medium (M), and small (S) droplets are 3.94, 3.15, and $2.29 \mathrm{~mm}$ for water droplets, and 3.08, 2.47, and $1.83 \mathrm{~mm}$ for surfactant droplets, respectively. The droplet speed is $v=2.4 \mathrm{~m} / \mathrm{s}$, and the film thickness is $h=0.3 \mathrm{~mm}$.

\section{Scaling relationship for the crown}

As shown above, the propagation of the crown during the impact process depends on a number of parameters, such as the impact speed, droplet size, and the average film thickness. It will be instructive to obtain a scaling relationship reflecting the influence of the effects of these parameters. For the impact of a droplet on a liquid film of the same fluid, Yarin and Weiss ${ }^{48}$ proposed a scaling for the propagation of the crown location given by

$$
\frac{d_{\text {crown }}}{d}=2\left(\frac{2}{3}\right)^{1 / 4} \frac{v^{1 / 2}}{d^{1 / 4} h^{1 / 4}}\left(t-t_{0}\right)^{1 / 2},
$$


where $t_{0}$ is a shifted time obtained via fitting of the experimental data. For their own experimental data ${ }^{48}$ there is an overestimation of the crown diameter by about $15 \%$, and for the experimental data by Levin and Hobbs, ${ }^{49}$ this overestimation is about $11 \sim 19 \%$. In Figure 10, we plot the crown rim diameter normalised by the droplet diameter and identify $t_{0}$ as the initial contact time of the droplet with the liquid film. As can be seen from Figure 10b, all the curves collapse approximately onto a straight line. The gradient of the straight line was used as a coefficient for the new scaling relationship of the crown rim,

$$
\frac{d_{\text {crown-rim }}}{d}=A \times 2\left(\frac{2}{3}\right)^{1 / 4} \frac{v^{1 / 2}}{d^{1 / 4} h^{1 / 4}}\left(t-t_{0}\right)^{1 / 2},
$$

where the coefficient $A=0.71$ was obtained by averaging the gradient of the lines obtained by least-square method.

Considering the additional complexity in the impact process brought about by the presence of surfactant, the relationship given by Eq. (2) provides a reasonable approximation of the temporal evolution of the location of the crown over a large fraction of its lifetime. Eq. (2) does not contain the viscosity and surface tension since it captures the dynamics during the formation and propagation of the crown when inertia is important. As the crown propagates outwards, its propagation speed reduces leading to a reduction in the contribution of inertial effects relative to that of surface tension and the viscosity, which become more dominant during the latter stages of the flow.

\section{Conclusions}

The impact of droplets on liquid film has been studied and the effects of surfactant and its difference in concentration between the droplet and the film have been analysed. Four surfactant configurations have been considered, namely water droplets on water films, water droplets on surfactant films, surfactant droplets on surfactant films, and surfactant droplets on water films. The effects of the droplet speed, the droplet size, and the film thickness have been considered. The flow pattern in 

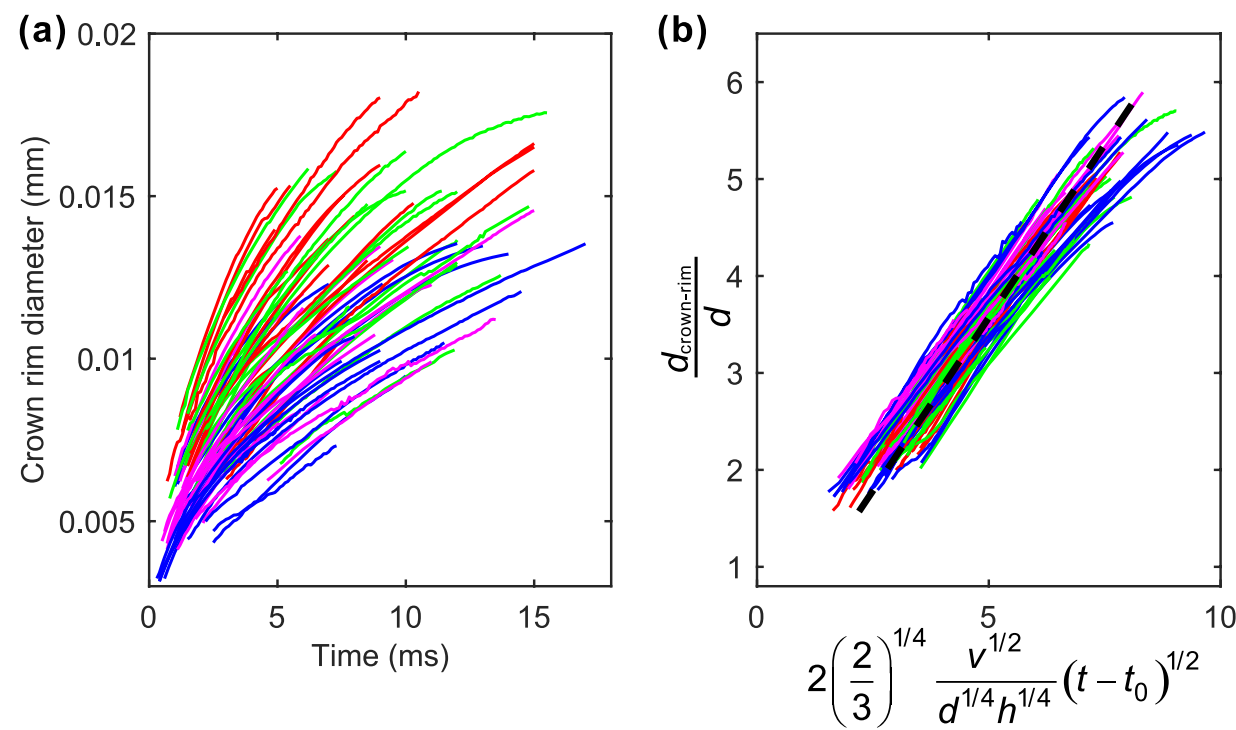

Figure 10: (a) Diameter of the rim of the crown for droplet impact on liquid films. (b) The dimensionless diameter of the crown $\operatorname{rim} d_{\text {crown-rim }} / d$. The curves collapse approximately onto a straight line with a gradient of $A=0.71$ (black dashed line). The colours of the lines indicate the surfactant configuration, red: water-water, green: water-surfactant, blue: surfactant-surfactant, magenta: surfactant-water. There are 70 curves in total representing 70 impact processes.

the liquid film has been revealed by using dye-tracing in the droplet phase. The results showed that the presence of surfactant in either phase can significantly alter the impact process. The film dye patterns form concentric circles and flower-shaped structures at low and high droplet Weber numbers, respectively. During the impact of surfactant droplets on water films, a strong radial flow is produced by the surface tension gradient due to the Marangoni effect, which may even lead to dewetting of the liquid film on the substrate. During the impact of water droplets on surfactant films, the flow in the liquid film is stabilized by the surfactant, and may inhibit flower patterns formed during the impact of water droplets on water films. The surfactant also alters the propagation of the capillary waves, the evolution of the crown, and the formation of secondary droplets.

This study will deepen our understanding of the physics involved in droplet impact processes and the role of surfactants in droplet dynamics. Although the effect of surfactants during the impact process begins to be understood, many questions remain unexplored. Measurements of the full flow field and numerical simulations of the impact process will provide new insights into the problem. In addition, the current study uses SDS, which is an anionic surfactant. Investigation 
of the effect of different types of surfactants, such as nonionic, anionic, and cationic surfactants, could be beneficial to understand the fundamental transport mechanisms of surfactants or ions at micro- or nanoscales.

\section{Acknowledgement}

We would like to acknowledge the support of the Engineering and Physical Sciences Research Council, UK, through the Programme Grant, MEMPHIS (EP/K003976/1) and National Natural Science Foundation of China (Grant No. 51676137).

\section{Supporting Information Available}

The following files are available free of charge.

- Video 1-figure3a.avi: Video clip for Figure 3a.

- Video 2-figure3b.avi: Video clip for Figure 3b.

- Video 3-figure3c.avi: Video clip for Figure 3c.

- Video 4-figure3d.avi: Video clip for Figure 3d.

- Video 5-figure6d.avi: Video clip for Figure 6d.

- Video 6-figure6i.avi: Video clip for Figure 6i.

This material is available free of charge via the Internet at http://pubs a a s org/.

\section{References}

(1) Josserand, C.; Thoroddsen, S. Drop impact on a solid surface. Annu. Rev. Fluid. Mech. 2016, 48, 365-391.

(2) Rein, M. Phenomena of liquid drop impact on solid and liquid surfaces. Fluid Dyn. Res. 1993, 12, 61-93. 
(3) Yarin, A. L. Drop impact dynamics: Splashing, spreading, receding, bouncingdots. Annu. Rev. Fluid. Mech. 2005, 38, 159-192.

(4) Deegan, R. D.; Brunet, P.; Eggers, J. Complexities of splashing. Nonlinearity 2007, 21, C1.

(5) Bird, J. C.; Tsai, S. S. H.; Stone, H. A. Inclined to splash: triggering and inhibiting a splash with tangential velocity. New J. Phys. 2009, 11, 063017.

(6) de Ruiter, J.; Oh, J.; van den Ende, D.; Mugele, F. Dynamics of collapse of air films in drop impact. Phys. Rev. Lett. 2012, 108, 074505.

(7) Driscoll, M. M.; Nagel, S. R. Ultrafast interference imaging of air in splashing dynamics. Phys. Rev. Lett. 2011, 107, 154502.

(8) Thoraval, M.-J.; Takehara, K.; Etoh, T. G.; Thoroddsen, S. T. Drop impact entrapment of bubble rings. J. Fluid Mech. 2013, 724, 234-258.

(9) Tran, T.; de Maleprade, H.; Sun, C.; Lohse, D. Air entrainment during impact of droplets on liquid surfaces. J. Fluid Mech. 2013, 726, R3.

(10) Gier, S.; Dorbolo, S.; Terwagne, D.; Vandewalle, N.; Wagner, C. Bouncing of polymeric droplets on liquid interfaces. Phys. Rev. E 2012, 86, 066314.

(11) Che, Z.; Deygas, A.; Matar, O. K. Impact of droplets on inclined flowing liquid films. Phys. Rev. E 2015, 92, 023032.

(12) Gao, X.; Li, R. Impact of a single drop on a flowing liquid film. Phys. Rev. E 2015, 92, 053005.

(13) Tran, T.; Staat, H. J. J.; Prosperetti, A.; Sun, C.; Lohse, D. Drop impact on superheated surfaces. Phys. Rev. Lett. 2012, 108, 036101.

(14) Xu, L.; Zhang, W. W.; Nagel, S. R. Drop splashing on a dry smooth surface. Phys. Rev. Lett. 2005, 94, 184505. 
(15) Hao, C.; Li, J.; Liu, Y.; Zhou, X.; Liu, Y.; Liu, R.; Che, L.; Zhou, W.; Sun, D.; Li, L. Superhydrophobic-like tunable droplet bouncing on slippery liquid interfaces. Nat. Commun. 2015, 6, 7986.

(16) Pan, K. L.; Law, C. K. Dynamics of droplet-film collision. J. Colloid Interface Sci. 2007, 587, $1-22$.

(17) Blanchette, F.; Bigioni, T. P. Partial coalescence of drops at liquid interfaces. Nat. Phys. 2006, 2, 254-257.

(18) Gilet, T.; Mulleners, K.; Lecomte, J. P.; Vandewalle, N.; Dorbolo, S. Critical parameters for the partial coalescence of a droplet. Phys. Rev. E 2007, 75, 036303-036303.

(19) Thoroddsen, S.; Takehara, K. The coalescence cascade of a drop. Phys. Fluids 2000, 12, $1265-1267$.

(20) Agbaglah, G.; Deegan, R. Growth and instability of the liquid rim in the crown splash regime. J. Fluid Mech. 2014, 752, 485-496.

(21) Mukherjee, S.; Abraham, J. Crown behavior in drop impact on wet walls. Phys. Fluids 2007, $19,052103$.

(22) Zhang, L. V.; Brunet, P.; Eggers, J.; Deegan, R. D. Wavelength selection in the crown splash. Phys. Fluids 2010, 22, 122105-122105.

(23) Thoraval, M. J.; Takehara, K.; Etoh, T. G.; Popinet, S.; Ray, P.; Josserand, C.; Zaleski, S.; Thoroddsen, S. T. von Kármán vortex street within an impacting drop. Phys. Rev. Lett. 2012, $108,264506-264506$.

(24) Thoroddsen, S. T.; Takehara, K.; Etoh, T. G. Micro-splashing by drop impacts. J. Fluid Mech. 2012, 706, 560-570.

(25) Howison, S. D.; Ockendon, J. R.; Oliver, J. M.; Purvis, R.; Smith, F. T. Droplet impact on a thin fluid layer. J. Fluid Mech. 2005, 542, 1-23. 
(26) Josserand, C.; Zaleski, S. Droplet splashing on a thin liquid film. Phys. Fluids 2003, 15, $1650-1657$.

(27) Lagubeau, G.; Fontelos, M. A.; Josserand, C.; Maurel, A.; Pagneux, V.; Petitjeans, P. Flower patterns in drop impact on thin liquid films. Phys. Rev. Lett. 2010, 105, 184503.

(28) Purvis, R.; Smith, F. T. Droplet impact on water layers: Post-impact analysis and computations. Philos. Trans. A Math. Phys. Eng. Sci. 2005, 363, 1209-1221.

(29) Tang, X.; Saha, A.; Law, C. K.; Sun, C. Nonmonotonic response of drop impacting on liquid film: Mechanism and scaling. Soft Matter 2016, 12, 4521-4529.

(30) van Hinsberg, N. P.; Budakli, M.; Göhler, S.; Berberović, E.; Roisman, I. V.; GambaryanRoisman, T.; Tropea, C.; Stephan, P. Dynamics of the cavity and the surface film for impingements of single drops on liquid films of various thicknesses. J. Colloid Interface Sci. 2010, 350, 336-343.

(31) Wang, A. B.; Chen, C. C. Splashing impact of a single drop onto very thin liquid films. Phys. Fluids 2000, 12, 2155-2158.

(32) Aboud, D. G. K.; Kietzig, A.-M. Splashing Threshold of Oblique Droplet Impacts on Surfaces of Various Wettability. Langmuir 2015, 31, 10100-10111.

(33) Zhang, R.; Hao, P.; He, F. Drop Impact on Oblique Superhydrophobic Surfaces with Two-Tier Roughness. Langmuir 2017, 33, 3556-3567.

(34) Thoroddsen, S. T.; Etoh, T. G.; Takehara, K. High-speed imaging of drops and bubbles. Annu. Rev. Fluid. Mech. 2008, 40, 257-285.

(35) Zhang, L. V.; Toole, J.; Fezzaa, K.; Deegan, R. D. Evolution of the ejecta sheet from the impact of a drop with a deep pool. J. Fluid Mech. 2012, 690, 5-15. 
(36) Hann, D. B.; Cherdantsev, A. V.; Mitchell, A.; McCarthy, I. N.; Hewakandamby, B. N.; Simmons, K. A study of droplet impact on static films using the BB-LIF technique. Exp. Fluids 2016, 57, 46.

(37) Frommhold, P. E.; Mettin, R.; Ohl, C.-D. Height-resolved velocity measurement of the boundary flow during liquid impact on dry and wetted solid substrates. Exp. Fluids 2015, $56,1-17$.

(38) Aytouna, M.; Bartolo, D.; Wegdam, G.; Bonn, D.; Rafaï, S. Impact dynamics of surfactant laden drops: Dynamic surface tension effects. Exp. Fluids 2010, 48, 49-57.

(39) Gatne, K. P.; Jog, M. A.; Manglik, R. M. Surfactant-induced modification of low Weber number droplet impact dynamics. Langmuir 2009, 25, 8122-8130.

(40) Nowak, E.; Kovalchuk, N. M.; Che, Z.; Simmons, M. J. Effect of surfactant concentration and viscosity of outer phase during the coalescence of a surfactant-laden drop with a surfactantfree drop. Colloids Surf., A 2016, 505, 124-131.

(41) Blanchette, F.; Bigioni, T. P. Dynamics of drop coalescence at fluid interfaces. J. Fluid Mech. 2009, 620, 333-333.

(42) Martin, D. W.; Blanchette, F. Simulations of surfactant effects on the dynamics of coalescing drops and bubbles. Phys. Fluids 2015, 27, 012103.

(43) Stoker, J. J. Water Waves: The mathematical Theory with Applications; John Wiley \& Sons, 2011; Vol. 36.

(44) Jensen, O. E.; Grotberg, J. B. Insoluble surfactant spreading on a thin viscous film: shock evolution and film rupture. J. Fluid Mech. 1992, 240, 259-288.

(45) Shen, H.; Hartland, S. Effect of interfacial concentration gradients of insoluble surfactants on local film-thinning. J. Colloid Interface Sci. 1994, 167, 94-103. 
(46) Yeo, L. Y.; Matar, O. K.; de Ortiz, E. S. P.; Hewitt, G. F. The dynamics of Marangoni-driven local film drainage between two drops. J. Colloid Interface Sci. 2001, 241, 233-247.

(47) Tan, C. S.; Gee, M. L.; Stevens, G. W. Optically profiling a draining aqueous film confined between an oil droplet and a solid surface: Effect of nonionic surfactant. Langmuir 2003, 19, $7911-7918$.

(48) Yarin, A. L.; Weiss, D. A. Impact of drops on solid surfaces: Self-similar capillary waves, and splashing as a new type of kinematic discontinuity. J. Fluid Mech. 1995, 283, 141-173.

(49) Levin, Z.; Hobbs, P. V. Splashing of water drops on solid and wetted surfaces: Hydrodynamics and charge separation. Philos. Trans. A Math. Phys. Eng. Sci. 1971, 269, 555-585. 


\section{Graphical TOC Entry}

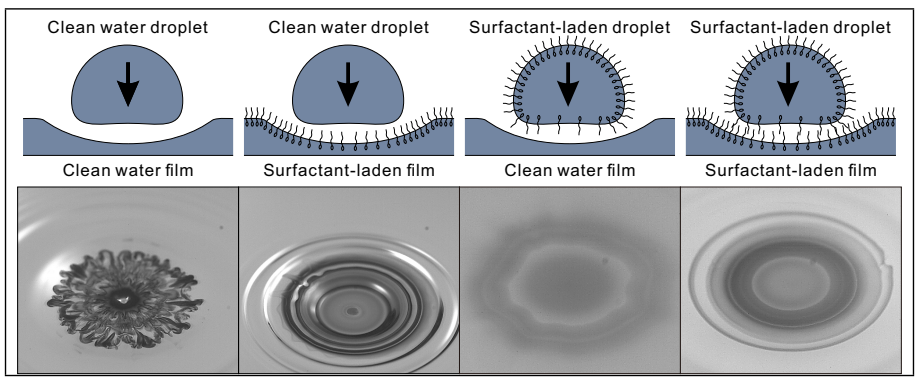

